\title{
Tracking the Source of Quantitative Knowledge in Neuroscience: A Neuroinformatics Role for Computational Models
}

\author{
John L. Baker • Giorgio A. Ascoli
}

Published online: 8 February 2011

(C) Springer Science+Business Media, LLC 2011

A significant benefit of computational models is that they mandate the explicit and quantitative account of all variables involved in the scientific problem being investigated. ${ }^{1}$ When a simulation is launched, the software program reads a series of parameter values that are assigned by the investigator. In compartmental models of neuronal electrophysiology, these parameters might correspond to membrane capacitances, axial resistances, synaptic conductances, reversal potentials, etc. for various parts of the neuron. In a model of neural network dynamics, parameters might include the interconnectivity, activation threshold, and input/output relations of the nodes or units. Other models will require biophysical parameters (and corresponding values) appropriate for the scale, domain, and scope of investigation. A developmental model of dendritic growth might use filopodia elongation and retraction rates, whereas a second messenger signaling model could instead use kinetic constants for each enzymatic step of the metabotropic cascade.

How are parameter values typically selected in computational neuroscience models? In experimentally driven approaches, a key component of the research design consists of an extensive literature search to determine suitable values or appropriate ranges for each parameter of the model. In a minority of cases, a direct measurement is available for a given parameter. More often, other related biophysical observables are reported, which can be employed to indirectly constrain the parameter in question. When even indirect measures are not available, the investigator may opt to leave

${ }^{1}$ Ascoli GA (2003) From data to knowledge. Neuroinformatics. 1(2):145-7.

J. L. Baker · G. A. Ascoli $(\bowtie)$

Center for Neural Informatics, Structures, \& Plasticity,

and Molecular Neuroscience Department,

Krasnow Institute for Advanced Study,

MS2A1 George Mason University,

Fairfax, VA 22030, USA

e-mail: ascoli@gmu.edu the "orphan" parameter open. In this scenario, a broad range of unconstrained values is tried in a number of otherwise identical simulations, and the results are monitored to reproduce or match some desired "emergent" property. Open parameter searches are usually considered a last resort because they increase the degrees of freedom in the model, limiting its predictive power and usefulness. Such searches are often time consuming, computationally intensive, and inconclusive.

Inferring parameter values from indirect measures encompasses a variety of diverse scenarios depending on how "related" the available empirical data are to the target parameter. This exercise, in fact, constitutes a continuum between the availability of direct measurements and the need for open searches to fit an observable result. In most circumstances, however, parameter value selection represents a critically delicate and labor intensive process in computational neuroscience. It may therefore be useful to develop of a database of quantitative neuroscience measures used as parameter values in computational models. Such an archive would provide an invaluable resource for reuse, while at once crucially documenting the details of parameter value selection. Each entry in the database would consist of a parameter definition and value(s) with associated metadata, including how the value was derived (direct, indirect, fitting), the literature reference(s) reporting the underlying measurements, and the specifics of the model that incorporates the parameter.

A database of model parameter values would exemplify a specific application of the notion of data publishing ${ }^{2}$ while complementing existing effort linking model sharing with literature mining ${ }^{3}$. Many difficulties would need to be

\footnotetext{
${ }^{2}$ De Schutter E (2010) Data publishing and scientific journals: the future of the scientific paper in a world of shared data. Neuroinformatics. 8(3):151-3.

${ }^{3}$ Davison AP, Morse TM, Migliore M, Shepherd GM, Hines ML (2004) Semi-automated population of an online database of neuronal models (ModelDB) with citation information, using PubMed for validation. Neuroinformatics. 2(3):327-32.
} 
overcome before putting such a vision into practice, not the least of which is creating appropriate ontologies to describe experiments, models ${ }^{4}$ and neuroscience metadata at large, which are still in their embryonic stages ${ }^{5}$. Yet the successful implementation of such a database, and its integration with relevant neuroinformatics tools ${ }^{6}$ would allow a much more efficient, powerful, and reproducible use of computational modeling in neuroscience. Most important would be the establishment of a direct and bidirectional link between sources and utilization of quantitative neuroscience data.

More generally, if appropriately improved and augmented, citation counts could become valuable for assessing the mutual value of neuroscience models and experiments. The present system of counting citations, even when enhanced by eigenfactor methods ${ }^{7}$ fails to reflect the ideal interaction between neuroscience models and experiments. Not only do model papers refer to experimental papers far more than the other way around, but references themselves provide little insight into how information from one type of research is used in the other. Modelers often cite a whole group of similar experiments even when only one of them has provided parameters for the simulations. Experimental papers typically cite an older but well known theory in their introduction, but usually only to provide a context, independent of any attempt to confirm or contradict the theory.

It is very challenging to objectively value models in intrinsic terms, such as quality of construction, consistency with experimental work, precision and clarity of results, or suggestions for further experiments. However, it would be quite informative to track how many times a model or separable pieces of the model are reused, for example, a particular ion channel formulation introduced in a model and adopted for a separate model, or sets of parameter values found indirectly or by best fitting. With a Google-style valuation process, the original source could then be credited in proportion to the value of the resulting new models and frequency of reuse.

Similarly, when a specific experimental result (e.g., a measured time constant) is directly or indirectly used in a model, the credit should go with the use. Evaluating experimental work on the basis of its contribution to models could be revealing and would encourage experimenters to report data in a form that facilitates reuse. This system would also require modelers to be specific about their sources (as discussed above), thus improving accuracy all around. If models yield experimentally testable predictions, there should be a way to keep track of which predictions were tested and what the results were. Both aspects should count in determining both the intrinsic value of the model itself and the experiments upon which it is based. A proper credit assignment scheme would promote experimental tests of high-value models.

To make this a closed system, models could be valued in terms of their use, both in new experiments and new models, and experiments could be valued in terms of how they contribute to both new models as data sources and old models as tests. Experiments can also contribute to further experiments through typically semi-quantitative estimates often found in discussion sections. Ideally, experiments and models would ultimately lead to progress outside of pure science such as new drugs or therapies, so those dimensions need to count also. Many radical advances are also coupled with improved methods. Similarly providing valuations for methods that are based on the impact of their subsequent usage would raise the profile of methods innovation. At least this is one area where citations might be a relatively trustworthy way of measuring reuse.

Unfortunately, working out robust metrics for such a scheme could be difficult. The most straightforward scheme would be based on simple reuse, but even that might be ill-defined when experimental data are used in an approximate sense or when parts of a model are adapted rather than adopted. Same-author reuse would require separate accounting. Statistical schemes exist to assess various aspects of information use when fitting complex models to data. Yet, a broadly acceptable metric spanning a wide range of current and future needs is still missing. A database that tracks who is reusing what and how could be an interesting step forward.

\footnotetext{
${ }^{4}$ Cannon RC, Gewaltig MO, Gleeson P, Bhalla US, Cornelis H, Hines ML, Howell FW, Muller E, Stiles JR, Wils S, De Schutter E (2007) Interoperability of neuroscience modeling software: current status and future directions. Neuroinformatics. 5(2):127-38.

${ }^{5}$ Zaveri A, Cofiel L, Shah J, Pradhan S, Chan E, Dameron O, Pietrobon R, Ang BT (2010) Achieving high research reporting quality through the use of computational ontologies. Neuroinformatics. 8(4):261-71.

${ }^{6}$ Marenco L, Wang R, Shepherd GM, Miller PL (2010) The NIF DISCO Framework: facilitating automated integration of neuroscience content on the web. Neuroinformatics. 8(2):101-12.

${ }^{7}$ Rizkallah J, Sin DD (2010) Integrative approach to quality assessment of medical journals using impact factor, eigenfactor, and article influence scores. PLoS One. 5(4):e10204.
} 